\title{
Evaluation of an accelerometer-based device to monitor compliance on patients wearing medical compression stockings
}

\section{E Grenier ${ }^{1}$, D Rastel $^{2}$, C Chaigneau ${ }^{1}$}

${ }^{1}$ SIGVARIS, Z.I. Sud d'Andrézieux, rue Barthélémy Thimonnier, BP 60223, 42170 Saint-Just Saint-Rambert, France

${ }^{2}$ Vascular physician, responsible for medical device vigilance, SELURL Philangio, 30 place Louis Jouvet, 38100

Grenoble, France

submitted: Jul 16, 2018, accepted: Nov 3, 2018, EPub Ahead of Print: Dec 9, 2018, published: Jul 7, 2019

Conflict of interest: D. Rastel is a private-sector vascular physician and non-exclusive consultant for medical textile manufacturer, SIGVARIS. E. Grenier and C. Chaigneau are employed by SIGVARIS in the respective functions of Research Associate and Head of Advanced Studies.

DOI: 10.24019/jtavr.51 - Corresponding author:Ing. Etienne Grenier, etienne.grenier@sigvaris.com

(C) 2018 Fondazione Vasculab impresa sociale ONLUS. All rights reserved.

\begin{abstract}
I- INTRODUCTION

Medical compression, for the most part employing textile methodology, constitutes the principal conservative treatment prescribed in the case of venous disorders. However, its role is subject to debate in the scientific community. Treatment is only effective if the orthosis is worn on a daily basis and clinical studies suffer from significant bias in relation to how patient compliance is measured.
\end{abstract}

II- AIMS

An electronic measurement device has been developed to measure patient compliance; it is based on movement detection by an accelerometer sensor. The aim of the present study is to compare objective data captured by the electronic device with wear times as reported by studied patients.

III- METHODS

The present study was conducted on 5 patients who were being treated for uncomplicated superficial venous insufficiency with a medical prescription for class 2 compression hose to be worn daily. Two pairs of currentlymarketed stockings were provided to the patients. One stocking of each pair was fitted with the electronic monitoring device. The patients were requested to wear their compression hose as normal and to note the dates and times of any incidents and any undesirable effects. IV- RESULTS
During the course of the study, the ten instrumented stockings captured 34 days that were representative of wear and 48 non-wear days. The absolute mean errors (and the standard deviation associated) calculated on the basis of the wear times reported by the patient and wear-times measured by the devices range between 5 (5) minutes and 83 (26) minutes, which corresponds to relative errors of between 1 $(0.9) \%$ and $19(7.1) \%$. For 27 days, the absolute difference between the subjective and the objective wear-times is less than 60 minutes. The results gathered by the sensors during non-wear days are 0 minute for 4 patients, representing 41 days of experimentation (85\%). On the other hand, one patient's devices incorrectly wrongly recorded average wear periods of 55 (55) minutes and 23 (16) minutes. No incidents or undesirable effects were reported by the patients.

$\mathrm{V}$ - CONCLUSION

The specially-developed electronic device is based on the principle of detection of phases of wear as registered by an accelerometer sensor. Integration of the system in an elastic compression stocking enables acquisition, analysis and interpretation of an acceleration signal thus providing objective monitoring of patient compliance as a function of the periods of wear and non-wear.

Keywords Medical compression stockings, compliance, accelerometer-based device 


\section{Introduction}

Compression therapy remains, at the present time, the cornerstone of lower limb venous disease treatment ${ }^{1,2}$. It decreases venous volume at rest, decreases ambulatory venous hypertension, increases muscle pump function and acts positively at the microcirculatory skin level ${ }^{3,4}$. Compression therapy is mainly textile in the form of bandages or stockings and its clinical results depends on different parameters such as the level of pressure, the cumulative pressure at rest and in movement, whether the textile is correctly applied on the leg or not ${ }^{5}$. So, among them, the wearing time is one of the greatest importance. A major bias in clinical studies on compression therapy is the compliance ${ }^{6}$. If compliance can be easily checked by nurses when bandages are used it is scarcely measured with stockings. When measured, it is through questionnaires that are firstly not validated in term of internal and external coherences and secondly self-administered by patients, consequently leading to biases ${ }^{7}$.

Patients at rest do not represent to a great extent a compliance problem since in most cases they are inpatients and hospital staffs regularly check the compression device. To solve the problem on active patients raised the idea to insert a sensor in the stockings. A first one « Thermotrack ${ }^{\circledR}$ » has been used in a restricted number of patients 7,8

Unfortunately, this electronic device based on temperature increase detection, cannot be used in certain climate. As a consequence, an electronic device based on motion detection with an accelerometer was developed. The objective of this work is to compare the objective results of the device to the wearing time reported by patients and then adapt its use to the compliance measurement of medical compression stockings (MCS) in active patients.

\section{Materials and methods}

\subsection{Electronic monitoring device}

\subsubsection{Technical characteristics and operating principle}

The electronic monitoring device is an electronic chip enabling assessment of the period of wear of a compression stocking. The system was developed by 3DOuest and Feichter Electronics (Lannion, France), specialists in electronic hardware and software design, on the basis of a SIGVARIS functional specification. It comprises a CR1616 button cell (Lithium 3V, 55mAh), with an autonomy of around four months in active mode and three key electronic components: - a three-dimensional accelerometer, a microcontroller and a memory. The accelerometer detects movement of the device embedded in the compression stocking and identifies the periods when the stocking is worn and when it is not worn.

Three parameters that are configurable when the electronic chips are initiated are designed to optimise detection of wear and non-wear periods. The cycle (or time interval) between two events can be set in a range going from 1 to 120 minute with a step of 1 minute. The movement detection threshold value was set at $0.3 \mathrm{G}$ and the accelerometer signal averaging time was 800 ms. These values were established by analysis of the data from experiments in real conditions of use and by integrating situations representative of product use in daily life (standing, sitting and lying down). The purpose of the parameters is to optimise detection sensitivity and the accuracy of data gathered relating to wear phases even in lengthy quasi-static situations and to minimise instances of false detection when the stocking is not being worn.

Wear data and the associated time-stamp are recorded at regular intervals in the embedded memory. Recording frequency can be set when the chip is initiated.

The 256-Kbits Electrically Erasable Programmable Read-Only Memory (EEPROM) can store data of wearing during 340 days with sampling every 15 minutes. The absolute maximum value of acceleration occurred during each period is stored on the first 7 bits of one byte. The acceleration value by triggering the motion detection is used to determine the wearing state (Yes or No). The advantage of this technology is that all recorded data is saved even in the absence of power. Consequently, there is no loss of data if the battery becomes discharged before the compliance data are extracted. Selection criteria for the electronic components and CR1616 microcontrollerembedded software were extremely stringent in order to meet energy-saving requirements and to ensure maximum device autonomy.

The electronic card lengths are $26 \times 16 \times 3 \mathrm{~mm}$. To ensure a perfect seal, the electronic card is included into a silicone casing which sizes lengths are $30 \times 20 \times 8 \mathrm{~mm}$ (Figure 1).

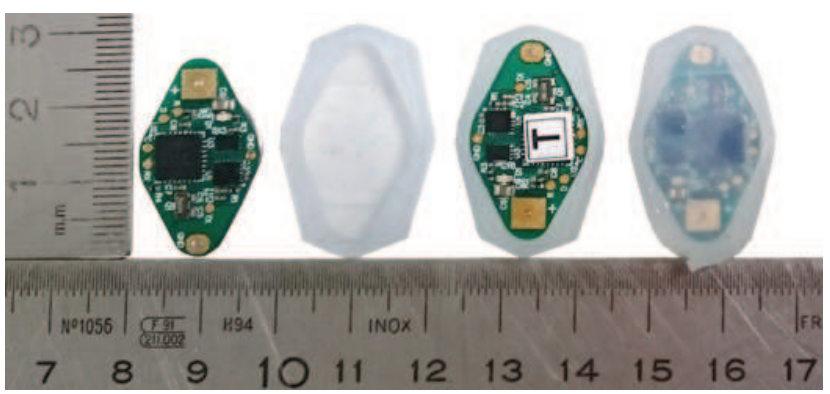

Figure 1 - From left to right: electronic monitoring chip, silicone base, device set inside the base, device sealed by potting silicone. 


\begin{tabular}{|lc|}
\hline Timestamp & Event detected \\
Tue Aug 10 19:12:34 & YES \\
Tue Aug 10 19:27:34 & YES \\
Tue Aug 10 19:42:34 & YES \\
Tue Aug 10 19:57:34 & YES \\
Tue Aug 10 20:12:34 & YES \\
Tue Aug 10 20:27:34 & YES \\
Tue Aug 10 20:42:34 & NO \\
Tue Aug 10 20:57:34 & NO \\
Tue Aug 10 21:12:34 & NO \\
Table I - Example of raw data extraction for a period of \\
two hours sampled every 15 minutes.
\end{tabular}

\begin{tabular}{|lcc|}
\hline Date & Wear time & $\begin{array}{c}\text { Wear time } \\
\text { (s) }\end{array}$ \\
Mon $5^{\text {th }}$ Sep & $12 \mathrm{~h} 30 \mathrm{~m}$ & 45000 \\
Tue $6^{\text {th }} \mathrm{Sep}$ & $7 \mathrm{~h} 15 \mathrm{~m}$ & 26100 \\
Wed $7^{\text {th }} \mathrm{Sep}$ & $0 \mathrm{~h} 0 \mathrm{~m}$ & 0 \\
Thu $8^{\text {th }} \mathrm{Sep}$ & $0 \mathrm{~h} 0 \mathrm{~m}$ & 0 \\
Fri $9^{\text {th }} \mathrm{Sep}$ & $11 \mathrm{~h} 15 \mathrm{~m}$ & 40500 \\
Sat $10^{\text {th }} \mathrm{Sep}$ & $0 \mathrm{~h} 0 \mathrm{~m}$ & 0 \\
Sun $11^{\text {th }} \mathrm{Sep}$ & $0 \mathrm{~h} 0 \mathrm{~m}$ & 0 \\
Mon $12^{\text {th }}$ Sep & $5 \mathrm{~h} 0 \mathrm{~m}$ & 18000 \\
Tue $13^{\text {th }}$ Sep & $5 \mathrm{~h} 30 \mathrm{~m}$ & 19800 \\
Wed 14 & 6h0m Sep & 21600 \\
& & \\
Table II - Example of a summary table generated by the \\
automatic software routine used to analyse data recorded \\
by electronic monitoring devices.
\end{tabular}

\subsubsection{Raw data extraction}

The data recorded in the monitoring device chip memory is extracted by means of dedicated software and a communication interface linked to a computer via an Universal Serial Bus (USB) connection. Communication between the electronic chip and the communication interface is via the electrical contact points. Raw data are displayed and can be saved to a spreadsheet file (.csv) in the software interface (Table I) for general analysis of patient compliance. This file contains all wear information (Yes / No) in the "Event detected» column, along with the relevant timestamp.

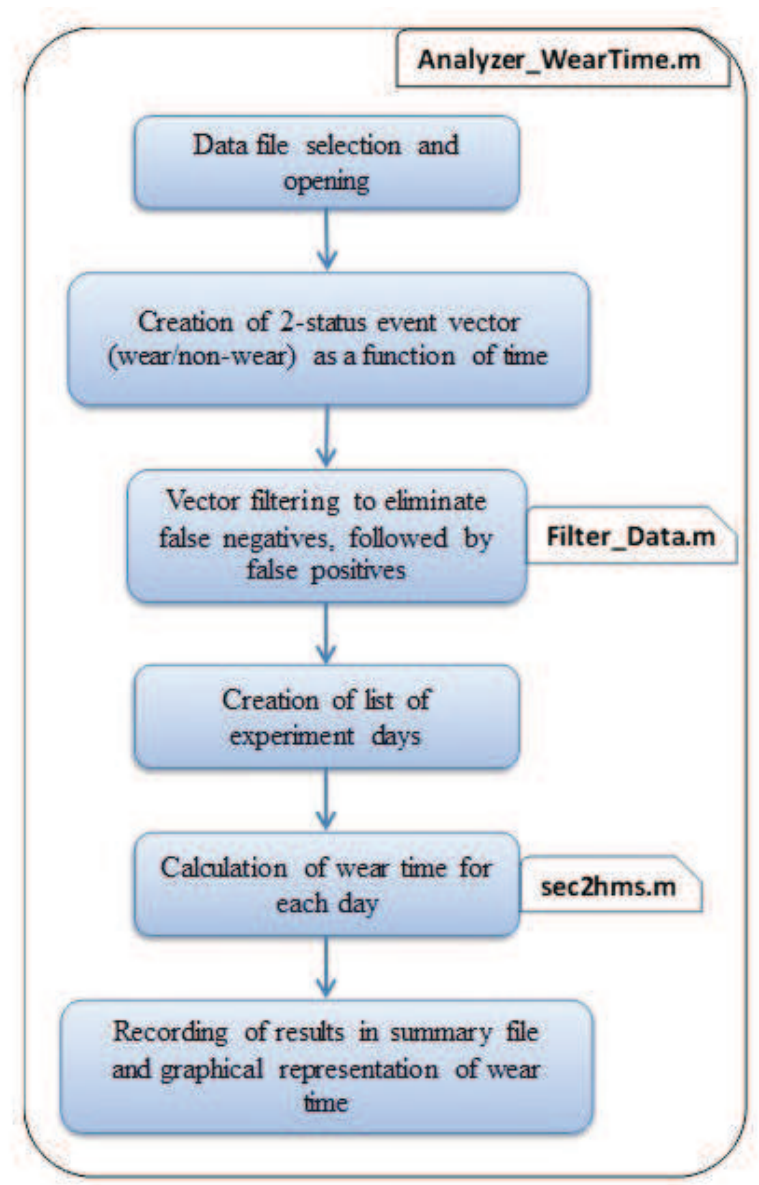

Figure 2 - Functional diagram for the automatic data processing algorithm

\subsubsection{Data processing, analysis and summary}

To facilitate raw data analysis, a data processing routine was developed in a programming environment (MATLAB $\AA$ ), comprising three functions. The main function handles sequential command execution and utilises the two other functions that are dedicated to filtering and data time conversion. Figure 2 illustrates the routine's algorithmic process.

"False negative" occurs when the device has not detected a real wear event. Conversely, a "false positive" occurs when the device has wrongly detected a wear event. Thus, by filtering the events, it is possible to eliminate cases of incorrect detection of wear or non-wear events. The digital filter was developed to take account of two consecutive periods functioning as a sliding window over the event vector. It acts as an averaging process (low-pass filter) for short and fast variations in the wear signal.

Based on the filtered signals, the programme generates and records two files: 


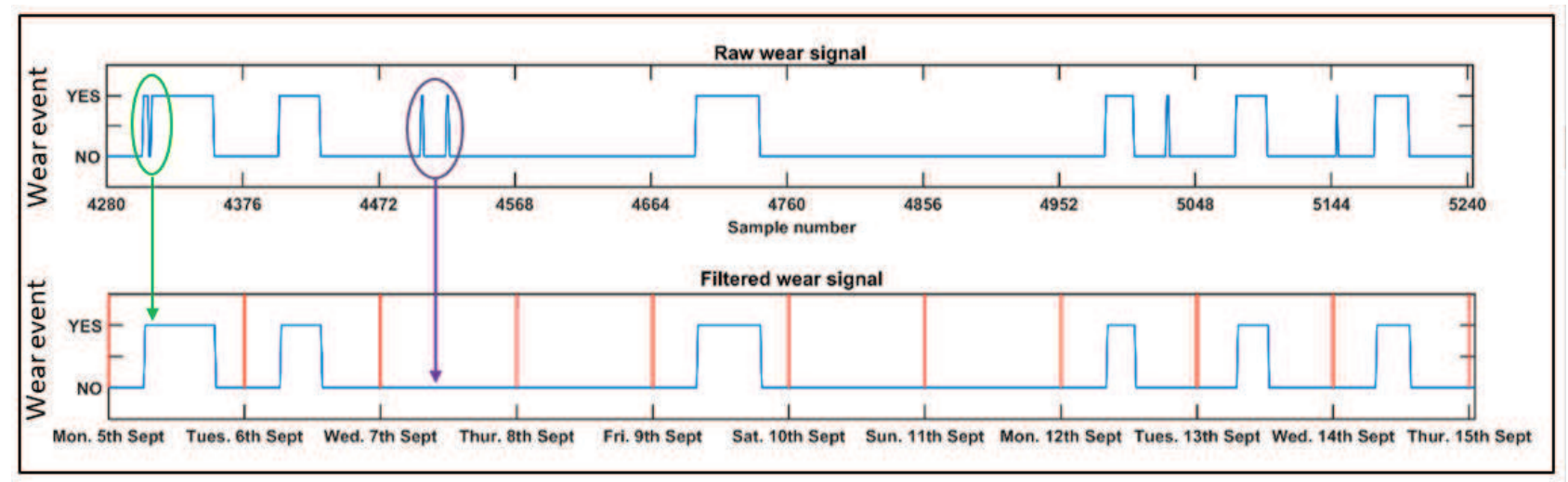

Figure 3 - Example of a graphical illustration of data monitored (YES/NO) obtained by the data extraction routine. Display of the effect of filtering in the elimination of false positives (purple circle) and false negatives (green circle). The red vertical lines represent the days of experimentation.

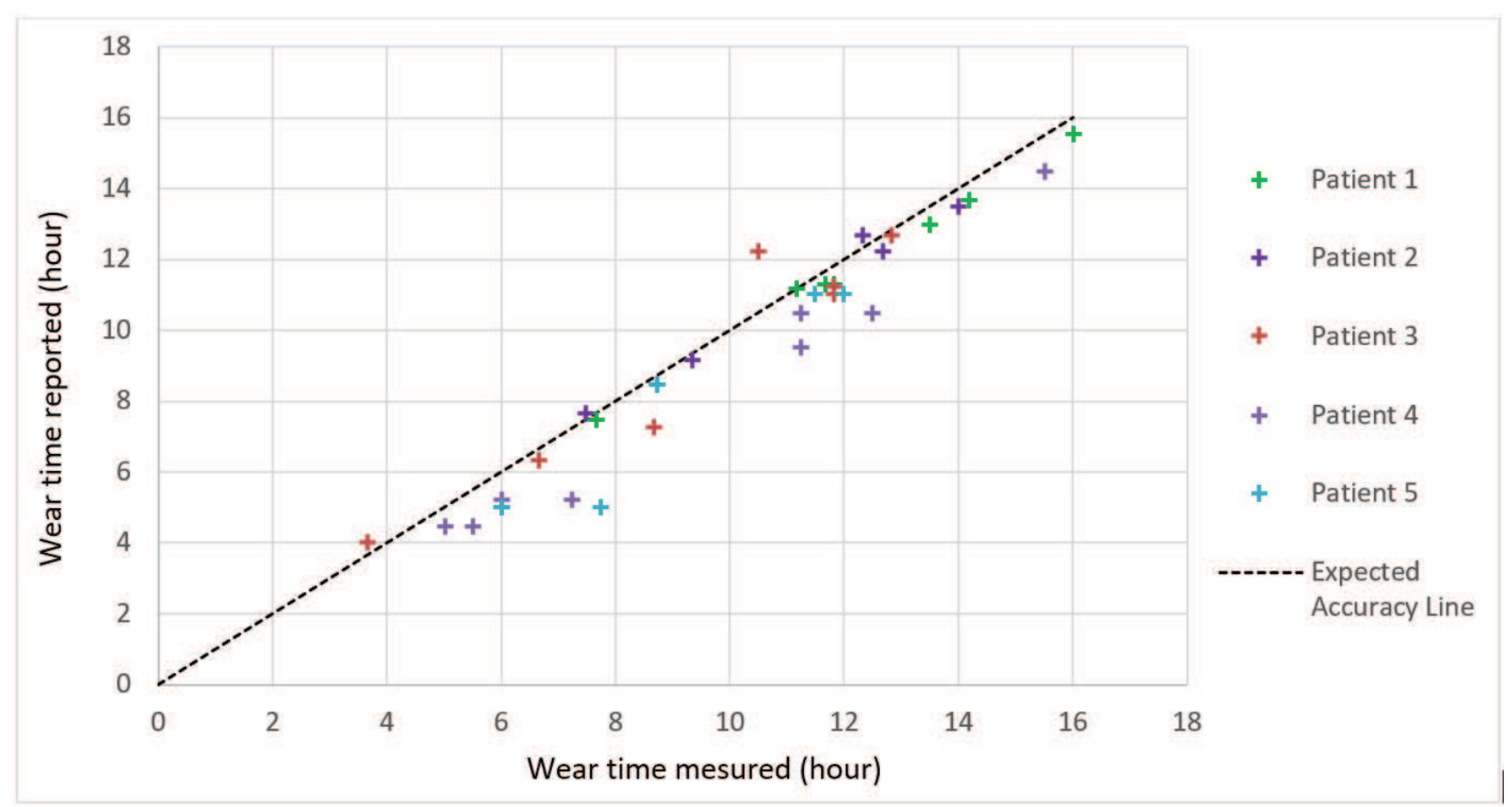

Figure 4 - Representation of wear times reported by the patients in comparison with wear times measured by the monitoring devices during the compression stocking wear days $(n=34)$

1. Daily wear time summary file (Table II). This file comprises three columns - event dates associated with wear times expressed in hours/minutes (unit commonly used) and seconds

2. Graphical representation of event vectors as a function of various types of filtering - raw signal and filtered signal of the MCS's wearing (Figure 3). This overview and visual representation are useful to quickly verify the raw signal recorded by the electronic device and the result of the filtering process. Also, it would be a quick and easy way to observe compliance data by the patient or the health professional.
Both Table II and Figure 3 document display the findings of the same experiment.

\subsubsection{Device integration in the medical compression stocking}

The electronic chip is protected against water ingress by silicone encapsulation (moulding and potting) which is resistant to hand- and machine-washing $\left(30^{\circ} \mathrm{C}\right.$ wash and $800 \mathrm{rpm}$ spin cycle). 
Testing was conducted to assess resistance to machine-washing. After thirty (or so) washes in a short cycle of 30 minutes at $30^{\circ} \mathrm{C}$, the electronic chips had not suffered any damage. The silicone envelopes were not damaged or torn and the electronic chips remained operational. This solution of silicone encapsulation can therefore be confirmed to guarantee water-tightness and device protection.

The device is inserted into the reverse side of the compression stocking and sewn in.

\subsection{Protocol}

Five patients receiving medical treatment for uncomplicated superficial venous insufficiency who had been prescribed class 2 compression stockings for daily wear (with a pressure of $15-20 \mathrm{mmHg}$ exerted on the ankle, AFNOR $^{9}$ standard G30 102 B), commercially-available and that was fitted with a sensor. MCS used in this study are socks composed of $50 \%$ Aquarius Polyamide / 27\% Elastane / 23\% Polyamide and $36 \%$ Polyamide / $16 \%$ Elastane / $48 \%$ Bamboo Viscose labelled URBAN and BAMBOU, respectively.

The patients gave their consent to participate in the study. As the device under test was to be ultimately used as part of clinical trials, this patient study was conducted as part of ANSM's (Agence Nationale de Sécurité Sanitaire du Médicament - National Agency for Safety of Health Products) medical device vigilance programme under the responsibility of the second author who is specifically responsible for elastic compression hose vigilance. Prior to testing, it was checked that the devices, once inserted into the stocking, had not altered the compression characteristics of the compression hose.

One stocking of the pair was fitted with the electronic monitoring device. Two pairs of "recording" stockings were distributed to the volunteers. The tests took place over an approximate period of one week. The patients were requested to wear their compression stocking as normal and to note date and time information relating to various wear events - donning and doffing, wash type (hand-wash or machine-wash), stocking handling (stretching, storage, etc.), and any undesirable effects.

The monitoring devices were configured for a 10 minute sampling and recording interval for patients 1,2 and 3 and 15 minutes for patients 4 and 5 .

\section{Results}

Stocking wear time data were extracted from the monitoring devices, processed and analysed in accordance with the methods outlined above. Each monitoring device was identified by the patient number and an alphabetical character to distinguish the stocking pairs allocated to the patient.

The results are presented separately for the days where the patients stated that they had worn their stocking and the days when they reported that they had not worn them. We consider that the reference wear times are those reported by the patients.

Over the course of the study, the ten instrumented stockings enabled us to capture 34 representative wear days and 48 days of non-wear. Figure 4 shows the wear times reported compared to the times measured by the monitoring devices and the theoretical straight line (dotted black line) shows a perfect relation between the two variables. It can be noted that in 30 out of 34 cases, the measured wear times are greater than the reported wear times.

Based on all the wear days, the averages and standard deviations were calculated for both the reported and the measured wear times. This data is used to determine the stocking wear time per day and after-the-event assessment of the patients' level of compliance (number of days per month and number of hours per day).

For example, if a wearing time of 10 hours had been recommended, it was observed that patient 1 was more compliant in terms of the average daily wear duration (723 (64) minutes/day for one pair and 710 (145) minutes/day for the other pair) compared to patient 5 (486 (149) minutes/ day and 405 (105) minutes /day). This observation is also confirmed by the wear times measured by the monitoring devices: 743 (71) and 732 (152) minutes/day for patient 1 and 552 (122) and 443 (83) minutes/day for patient 5.

For each wear day, the absolute and relative differences were also calculated on the basis of the wear times reported by the patients and the durations measured by the devices. The absolute mean errors between these two wear time types range between 5 (5) minutes and 83 (26) minutes, which corresponds to errors varying from 1 (0.9)\% and 19 (7.1)\% compared to the reported reference time. Table III summarises all reported and measured data and provides the results of associated comparisons. Both columns contained under each patient present the results from a device integrated in MCS. For example, D1a means Device integrated in the first pair of the patient 1 and D1b in the other pair.

For the entire period of wear, 34 days, eighteen show an absolute difference between the subjective and objective wear time of less than 30 minutes. This difference is between 30 and 60 minutes on 9 days and between 1 hour and 2 hours 45 minutes on 7 days (Figure 5).

Moreover, it is interesting to analyse the data measured by the monitoring devices during the periods when the stockings were not worn. The phenomenon known 
as "false negative" indicates incorrectly reports wear data and consequently leads to overestimation of the total wear duration.

In our experiment, according to the patients, the total number of non-wear days was 48. The results obtained from the monitoring devices are consistent with these non-wear phases as the wear durations are equal to 0 minutes for 4 patients out of 5, thus representing 41 days of experimentation $(85 \%)$. By contrast for the patient 1 , monitoring devices recorded an average wear period of 55 (55) minutes for pair $A$ and 23 (16) minutes for pair B, when in fact medical compression stockings were not worn (Table IV).

\begin{tabular}{|c|c|c|c|c|c|c|c|c|c|c|c|}
\hline & \multicolumn{2}{|c|}{ Patient 1} & \multicolumn{2}{|c|}{ Patient 2} & \multicolumn{2}{|c|}{ Patient 3} & \multicolumn{2}{|c|}{ Patient 4} & \multicolumn{2}{|c|}{ Patient 5} \\
\hline & & D1a & D1b & $\mathrm{D} 2 \mathrm{a}$ & $\mathrm{D} 2 \mathrm{~b}$ & D3a & D3b & D4a & $\mathrm{D} 4 \mathrm{~b}$ & D5a & D5b \\
\hline \multicolumn{2}{|c|}{$\begin{array}{c}\text { Experimental day day } \\
\text { considered }\end{array}$} & 3 & 4 & 3 & 2 & 4 & 3 & 6 & 2 & 5 & 2 \\
\hline Wear Time & Mean minute & 723 & 710 & 668 & 655 & 634 & 450 & 405 & 720 & 486 & 405 \\
\hline Reported & (std) & (64) & $(145)$ & (139) & (105) & (127) & (150) & $(150)$ & (150) & (149) & (105) \\
\hline Wear Time & Mean minute & 743 & 732 & 683 & 650 & 628 & 483 & 475 & 803 & 552 & 443 \\
\hline Measured & (std) & (71) & $(152)$ & (156) & $(90)$ & (114) & (176) & (158) & (128) & (122) & (83) \\
\hline \multirow{4}{*}{$\begin{array}{l}\text { Absolute } \\
\text { difference }\end{array}$} & Mean minute & 20 & 22 & 22 & 5 & 46 & 47 & 75 & 83 & 66 & 53 \\
\hline & & (13) & (8) & (10) & (5) & (29) & (26) & (30) & (26) & (40) & (8) \\
\hline & Max minute & 30 & 30 & 30 & 10 & 105 & 85 & 120 & 105 & 165 & 60 \\
\hline & Min minute & 0 & 10 & 0 & 0 & 10 & 20 & 30 & 60 & 15 & 45 \\
\hline $\begin{array}{l}\text { Relative } \\
\text { difference }\end{array}$ & $\begin{array}{c}\text { Mean } \\
\text { (std) }\end{array}$ & $\begin{array}{c}4 \\
(0.4)\end{array}$ & $\begin{array}{c}3 \\
(0.5)\end{array}$ & $\begin{array}{c}3 \\
(0.6)\end{array}$ & $\begin{array}{c}1 \\
(0.9)\end{array}$ & $\begin{array}{c}7 \\
(3.8)\end{array}$ & $\begin{array}{l}11 \\
(5.7)\end{array}$ & $\begin{array}{c}19 \\
(7.1)\end{array}$ & $\begin{array}{c}13 \\
(5.8)\end{array}$ & $\begin{array}{c}18 \\
(15.3)\end{array}$ & $\begin{array}{c}14 \\
(5.6)\end{array}$ \\
\hline
\end{tabular}

Table III - Summary of stocking wear times obtained from patient reports and from data for wear days as measured by the monitoring device. Dla means "device integrated in the first pair of the patient 1 and D1b in the other pair" and so on for the other patients.

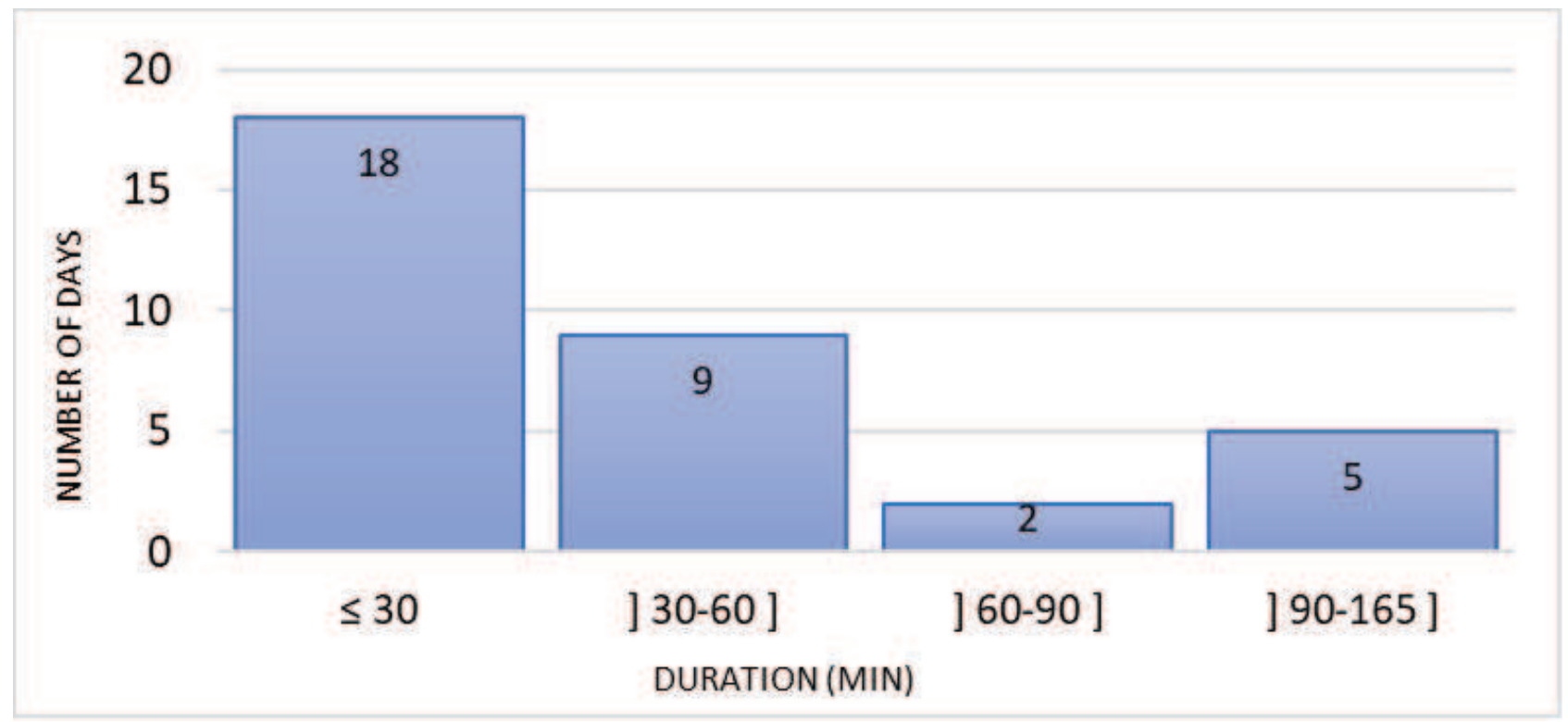

Figure 5 - Breakdown of absolute wear time differences between patient-reported data and data captured by the monitoring devices 


\begin{tabular}{|c|c|c|c|c|c|c|c|c|c|c|c|}
\hline & & Pat & nt 1 & Pati & nt 2 & Pati & nt 3 & Pat & nt 4 & Pat & nt 5 \\
\hline & & D1a & D1b & $\mathrm{D} 2 \mathrm{a}$ & $\mathrm{D} 2 \mathrm{~b}$ & D3a & D3b & $\mathrm{D} 4 \mathrm{a}$ & D4b & D5a & D5b \\
\hline Exper & $\begin{array}{l}\text { ntal day day } \\
\text { sidered }\end{array}$ & 4 & 3 & 4 & 5 & 3 & 4 & 4 & 8 & 5 & 8 \\
\hline $\begin{array}{l}\text { Absolute } \\
\text { difference }\end{array}$ & $\begin{array}{l}\text { Mean minute } \\
\text { (std) }\end{array}$ & $\begin{array}{c}55 \\
(55)\end{array}$ & $\begin{array}{c}23 \\
(16)\end{array}$ & $\begin{array}{c}0 \\
(0)\end{array}$ & $\begin{array}{c}0 \\
(0)\end{array}$ & $\begin{array}{c}0 \\
(0)\end{array}$ & $\begin{array}{c}0 \\
(0)\end{array}$ & $\begin{array}{c}0 \\
(0)\end{array}$ & $\begin{array}{c}0 \\
(0)\end{array}$ & $\begin{array}{c}0 \\
(0)\end{array}$ & $\begin{array}{c}0 \\
(0)\end{array}$ \\
\hline & Max minute & 130 & 40 & 0 & 0 & 0 & 0 & 0 & 0 & 0 & 0 \\
\hline & Min minute & 0 & 0 & 0 & 0 & 0 & 0 & 0 & 0 & 0 & 0 \\
\hline
\end{tabular}

\section{Discussion}

The principal outcome of the study is confirmation of the effectiveness of an electronic device to measure patient compliance with prescribed wear of medical compression stockings. During the study, measurements were recorded by an electronic chip using an accelerometer to detect movement of the device and also records the data at regular intervals.

The central aim of the system is to help in a greater extent a health practitioner to obtain data relating to the overall use of the prescribed compression hose. Despite the uncertainty of daily wear times stemming from the monitoring devices, thanks to the sensor it is possible to assess a patient's pattern of compliance, namely by taking into consideration the days when the compression stockings are not worn. Thus, what is the impact on the health practitioner's conclusions based on daily wear data that might include an error of one or two hours? Is currently our monitoring device sufficiently accurate to meet the requirements of compliance objectification or the level of compliance observed by the ultimate data user?

Under certain conditions of daily use, the electronic chips may incorrectly detect movement (and be confused with wear periods). These "false positives" may be due to different phases of use of the compression hose, such as, for example, washing, drying in the open air (wind effect), spare pair of stockings being carried in a handbag, stockings being displaced in a wardrobe, etc. Incorrect detections may then induce an error in the daily wear time results, the duration of which may vary as a function of the conditions of use. Conversely, when the compression stockings are effectively being worn, it is possible that the device does not detect any movement during periods when the lower limbs are immobile (when taking a siesta, or sitting down reading or watching TV). However, we have no means of evaluating said errors (over estimation or underestimation) measured by the monitoring devices in relation to usage criteria and to distinguish them between true periods of use.
The reliability of the results captured by the monitoring devices was assessed by comparison with the wear times reported by the patients. In spite of strict experimental conditions, it is possible that the reported compression stocking donning and doffing times are, to a certain extent, approximate, depending on the time lapse between the event and the moment the event is noted down. For example, it might be difficult to remember the exact time the compression stockings are donned if the wear times are noted down at the end of the day. The accuracy of this data may be questionable, which might, to a certain extent, explain the differences between the reported and measured periods of wear. This therefore introduces another period relating compression stocking wear time that is defined by the true wear duration.

During the experiment, the interval between each data capture of compression stocking wear was set at between 10 and 15 minutes. This temporal resolution could possibly be reduced to 5 minutes in order to minimise the error induced by false positives. The wear time results are very frequently overestimated by the monitoring devices in relation to the times reported by the patients. The overestimation is less than one hour for over three quarters of the wear days and the maximum overestimation was 2 hours 45 minutes. These variations can be explained by independent or successive washing, drying and storage phases that are wrongly considered to be wear phases, due to detection of stocking movement.

From a material point of view, the devices are encapsulated in a silicone case and it is not possible to check the functional status during the trial without destroying the device. To access recorded data or data in the course of recording, it is necessary to break into the compression hose and the case in order to retrieve the data via the communication interface between the chip and the computer. It would be preferable to be able to interrogate the monitoring device via a wireless connection (Bluetooth, WiFi, ZigBee, RFID, NFC, etc.). 
This functionality would not only enable us to verify the operational status of the electronic system but also to extract and display at regular intervals the data saved in the memory. Furthermore, the autonomy is not sufficient to enable evaluation of compliance over a 6 -month period (compression hose legal guarantee period); we will have to find solutions to overcome this difficulty. The device's compact design constitutes a major advantage, as there is no discomfort when the compression stockings are being worn. In a large-scale mass-production approach, power autonomy could be increased and the dimensions could be very significantly reduced by the use of specific, more powerful electronic components.

The device developed has been validated, contrary to other devices already in use. However, in the future, advancements in wear detection systems are likely to focus on physiological parameter acquisition (dermal impedance, capacitive proximity detection, etc.) that is modified solely by textile/lower limb interaction.

\section{Conclusion}

We have developed an electronic device based the detection of wear times by means of an accelerometer

\section{References}

1) Wittens C, Davies AH, Baekgaard R, Brohlom A, Cavezzi A, Chastanet S, et al. Management of Chronic Venous Disease. Clinical Practise Guidelines of the European Society for Vascular Society (ESVS). Eur J Vasc Endovasc Surg 2015; 49: 678-737.

2) Rabe E, Partsch H, Hafner J, et al. Indications for medical compression stockings in venous and lymphatic disorders: An evidence-based consensus statement. Phlebology 2017; doi: $\underline{10.1177 / 0268355516689631}$

3) Bergan JJ, Geert W, Schmid-Schönbein PD, et al. Chronic venous insufficiency. N Engl J Med 2006; 355: 488-98.

4) Partsch H. Mechanism and effects of compression therapy. In: The Vein Book. London: Elsevier; 2007, 103-109.

5) Partsch H. Compression therapy: clinical and experimental evidence. Ann Vasc Dis 2012; 5: 416-22. associated with an algorithm to process, analyse and summarise patient compliance reported on a daily basis. By embedding this system in an elastic compression stocking, we have demonstrated that it is possible to objectivise the wear and non-wear phases by acquisition, analysis and interpretation of an accelerometer sensor signal.

The trial consisted of a study conducted by 5 patients wearing medically-prescribed compression stockings in order to evaluate the reliability of the results delivered by the monitoring devices. We captured 34 days representative of compression stocking wear days and 48 non-wear days. In the course of the experiment, we have demonstrated that data relating to patient compliance with a medical prescription for compression stockings can be acquired and recorded over a period of several months without changing the patients' normal lifestyle. The difference between the wear times reported by the patients and the durations measured by the monitoring devices is less than 60 minutes for $79 \%$ of the wear days. The results of the monitoring devices also demonstrate the accuracy of the data in $85 \%$ of the days when the elastic compression stockings were not worn. These errors could result in uncertainty as to the actual daily wear durations but do not, however, have a major impact on objectivation of overall patient monitoring.

6) Ziaja D, Koce\#ak P, Chudek J, Ziaja K. Compliance with compression stockings in patients with chronic venous disorders. Phlebology. 2011; 26(8): 353-60

7) Allaert FA, Rastel D, Graissaguel A, Sion D, HamelDesnos C. Design and evaluation of the psychometric properties of a self-questionnaire on patient adherence to wearing elastic compression stockings. Phlebology. 2018 Jan 1:268355518762824. doi: $10.1177 / 0268355518762824$. Epub ahead of print]

8) Uhl JF, Benigni JP, Chahim M, Fréderic D. Prospective randomized controlled study of patient compliance in using a compression stocking: Importance of recommendations of the practitioner as a factor for better compliance. Phlebology. 2018; $33: 36-43$.

9) AFNOR, Association Française de NORmalisation. https:// www.afnor.org/en/ 